\title{
Transformaciones del Sistema de Salud Ecuatoriano
}

\author{
Transformation of health system in Ecuador \\ Wilson Giovanni Jiménez-Barbosa1* ${ }^{*}$ orcid.org/0000-0002-0467-0365 \\ María Luisa Granda-Kuffo² \\ Diana Margoth Ávila-Guzmán³ orcid.org/0000-0002-4459-028X \\ Leidy Johanna Cruz-Díaz ${ }^{4}$ orcid.org/0000-0001-9134-6063 \\ Julián Camilo Flórez-Parra ${ }^{5}$ orcid.org/0000-0002-9869-7867 \\ Luisa Silvana Mejíá orcid.org/0000-0002-7858-6596 \\ Diana Carolina Vargas-Suárez ${ }^{3}$ orcid.org/0000-0002-1168-1759
}

1. Universidad Jorge Tadeo Lozano. Bogotá, Colombia.

2. Escuela Superior Politécnica del Litoral, ESPOL, ESPAE Graduate School of Management, Campus Peñas Malecón 100 y Loja, P.O. Box 09-01-5863, Guayaquil, Ecuador

3. Hospital Vista Hermosa. Bogotá, Colombia.

4. Clínica Colsubsidio Calle 100. Bogotá, Colombia

5. Proyecto INPEC-CAPRECOM. Bogotá, Colombia

6. Certificar IPS. Bogotá, Colombia.

\section{Resumen}

Objetivo: Describir las transformaciones de la estructura del Sistema de Salud Ecuatoriano, teniendo en cuenta el contexto constitucional y normativo de dicho país, y reflexionar sobre el contexto histórico en que acaecieron y sus implicaciones en el bienestar de los ecuatorianos. Materiales y métodos: Se realizó una revisión bibliográfica, analizando la normatividad de Ecuador desde la Constitución de 1979, en donde se empieza hablar de la salud como un derecho, pasando por la Ley Orgánica de Salud, la Ley de Seguridad Social, entre otras, incluyendo la última reforma de la Constitución en 2008. Resultados: La transformación del Sistema de Salud del Ecuador es el resultado de la acción de fuerzas políticas y económicas tanto internas como externas que han afectado a este país a lo largo del periodo estudiado.

Palabras clave: Sistemas de salud; Ecuador; Constitución; Ley Orgánica. (Fuente: DeCS, Bireme).

\begin{abstract}
Objective: To describe the transformations of the structure of the health system in Ecuador, taking into account the constitutional and policy context of that country, and to reflect on the historical context in which it occurred and its implications for the welfare of the people from Ecuador. Materials and methods: A bibliographic review was made, beginning with the regulations of Ecuador since the Constitution of 1979, where health is considered as a right, passing by the Organic Law of Health, the Social Security Act, among others, including the last reform of the Constitution in 2008. Results: The transformation of the Health System of Ecuador is the result of the action of economic and political forces, both internal and external, that have affected this country throughout the studied period.
\end{abstract}

Keywords: Health systems; Ecuador; Constitution; organic law. (Source: DeCS, Bireme). 


\section{Introducción}

El Sistema de Salud ecuatoriano ha estado bajo constantes cambios, principalmente debido a las transformaciones estructurales del modelo económico y de desarrollo, que han estado ligadas a las tres últimas constituciones que han regido en el país, promulgadas en los años de 1979, 1998 y la nueva Constitución Política, aprobada a partir del año $2008^{1}$.

Cada cambio constitucional se ha correspondido con momentos críticos vividos en Ecuador, así la Constitución de 1979 se dio en el marco de la culminación de un periodo de dictadura militar, la de 1998 en el advenimiento de las políticas neoliberales y finalmente, la de 2008, en el surgimiento de un movimiento cívico como respuesta a la inestabilidad política que se presentó en la primera mitad del decenio del 2000.

Este movimiento cívico condujo al poder al economista Rafael Correa, quien logró consolidar unas mayorías populares y parlamentarias que le permitieron realizar profundas transformaciones en los ámbitos social, político y económico, que como resultado ha reconfigurado a Ecuador, al dejar atrás el modelo neoliberal de los 90 para constituirse en un país que podría calificarse como social demócrata al tomar el Estado un rol principal en la regulación de los mercados y en la garantía universal y equitativa de la provisión de derechos fundamentales como el de educación y salud.

En el sector salud estos cambios se expresan, en especial, en los artículos 32 y 34 de la Constitución de 2008. El artículo 32, no limita la salud, al buen estado físico y mental de una persona, sino que también, incluye otras áreas vinculadas con la política social tales como: servicios públicos, alimentación, educación, trabajo, seguridad social, cultura física, los ambientes sanos y todo lo que garantice o mejore las condiciones y la calidad de vida de las personas. Por su parte, en el artículo 34 la seguridad social se plantea como un derecho irrenunciable tanto para los individuos como para suplir las necesidades de las comunidades ${ }^{1}$.
A partir de este marco general, el presente artículo tiene como objetivo describir las transformaciones de la estructura del Sistema de Salud Ecuatoriano, teniendo en cuenta el contexto constitucional y normativo de dicho país, y reflexionar sobre el contexto histórico en que acaecieron y las implicaciones que tuvieron para el bienestar de los ecuatorianos.

\section{Materiales y métodos}

Esta investigación es de tipo cualitativo, descriptivo, soportada sobre revisiones bibliográficas adelantadas en bases de datos, tales como: Elsevier, SciELO, PubMed, Science Direct y páginas oficiales del Gobierno Ecuatoriano.

Para esta búsqueda se utilizaron como palabras clave: sistemas de salud, Ecuador, Constitución, Ley orgánica, como resultado se identificaron 40 documentos de los cuales se revisó el resumen y las principales conclusiones para determinar si la temática abordada correspondía con descripción y/o análisis del Sistema de Salud de Ecuador o aportaban a comprender su demografía, su sistema político y administrativo. Resultaron seleccionados 28 documentos que cumplían con los criterios definidos. La literatura acerca de la estructura y evolución del Sistema de Salud de Ecuador en revistas académicas es escasa y, por tanto, las principales fuentes encontradas fueron documentos oficiales, dentro de los que se destacan normas del corpus legal.

Estos documentos fueron leídos en toda su extensión y sus contenidos analizados, extrayéndose de ellos sus principales conceptos y categorías, los cuales fueron consolidados mediante fichas bibliográficas diseñadas por los autores. Finalmente, con los hallazgos de los documentos se procedió a estructurar el contenido de la investigación, partiendo de describir la demografía y estructura política de Ecuador para continuar con las particularidades del sistema. Para la discusión se recurrió a documentos de política pública internacional que sirvieran de marco de análisis comparativo entre 
la evolución histórica del Sistema de Salud Ecuatoriano y el contexto internacional, en especial el latinoamericano.

\section{Resultados}

Los resultados se presentan en tres acápites principales: el primero, en el que se realiza una descripción de Ecuador y de su división geopolítica; en el segundo, se refieren los cambios normativos que han impactado la garantía de la salud Ecuador y finalmente, en el tercero se narra la forma en que actualmente está estructurado el Sistema de Salud.

\section{Ecuador}

Ecuador es un Estado constitucional de derechos y justicia, social, democrático, soberano, independiente, unitario, intercultural, plurinacional y laico. Se organiza en forma de República y se gobierna de manera descentralizada. ${ }^{1}$ Posee un área de $283.561 \mathrm{~km}^{2}$ y una proyección de población de 16.278.844 personas, para el año 2015, según el Compendio Estadístico de 2013, de los cuales el 50,4\% son mujeres (8.216.234 habitantes) y el $49,5 \%$ son hombres (8.062.610 habitantes), que representa una densidad poblacional de 61 habitantes por kilómetro cuadrado² .

\section{Organización político- administrativo}

Según el artículo 242 de la Constitución Nacional de 2008, la República de Ecuador está organizada en regiones, provincias, cantones y parroquias rurales, las cuales se describen de menor a mayor jerarquía, a continuación ${ }^{1}$ :

1. Parroquia: División de menor jerarquía político-administrativa y se clasifica en rural y urbana. La parroquia rural es aquella que está distanciada de la ciudad, generalmente dedicada a las labores del campo y la urbana que se encuentra en cercanía a las grandes ciudades. En total en Ecuador existen 1149 parroquias (790 rurales y 359 urbanas).

2. Cantón: Conforma el segundo nivel de jerarquía político-administrativa. Está constituido por la unión de dos o más parroquias. En total en Ecuador existen 221 cantones.

3. Provincia: Unión de dos o más cantones, los cuales conforman el mayor nivel de jerarquía político-administrativa del Ecuador. En total hay 24 provincias, las cuales, se pueden agrupar para formar regiones autónomas, en consonancia con lo establecido constitucionalmente ${ }^{1}$.

\section{Niveles administrativos de planificación}

En el 2012, la Secretaría Nacional de Planificación y Desarrollo, SENPLADES, tenía como propósito lograr que el Estado prestara servicios más eficientes y acordes con las necesidades de la población, a partir de esto, mediante el Registro Oficial Edición Especial $\mathrm{N}^{\circ}$ 290 de 2012, se conformaron niveles administrativos de planificación: zonas, distritos $\mathrm{y}$ circuitos a nivel nacional ${ }^{3}$. Esta forma de organización pretende lograr una mejor identificación de necesidades y soluciones efectivas para la prestación de servicios públicos en el territorio. Esta conformación no implica eliminar las provincias, cantones o parroquias, es decir, estos niveles de gobierno conservan su autonomía y funciones.

Los niveles de planificación buscan contar con una oferta ideal de servicios en el territorio sustentado en un Estado planificado, descentralizado, articulado, equitativo, con mayor cobertura y calidad de servicios públicos ${ }^{3}$. Cabe mencionar que los distritos y circuitos se han aplicado prioritariamente a sectores como el social, seguridad, entre otros, aunque no todos los sectores están incluidos.

Según el Registro Oficial Edición Especial N² 290 de 2012, en la cual se publica la Reforma STDCGJ-RA-0088-2014 (Refórmese el Estatuto Orgánico la Secretaría Nacional de Planificación y Desarrollo) se establecieron como niveles de planificación los siguientes ${ }^{3}$ :

1. Zonas: Están conformadas por provincias vecinas. Desde este nivel se coordina estratégicamente las entidades del sector público. Existen 9 zonas de planificación 
constituidas por distritos, las cuales se relacionan a continuación:

Zona 1: Esmeraldas, Imbabura, Carchi, Sucumbíos.

Zona 2: Pichincha (excepto Quito), Napo, Orellana.

Zona 3: Cotopaxi, Tungurahua, Chimborazo, Pastaza.

Zona 4: Manabí, Santo Domingo de los Tsáchilas.

Zona 5: Santa Elena, Guayas (excepto Guayaquil, Samborondón y Durán), Bolívar, Los Ríos y Galápagos.

Zona 6: Cañar, Azuay, Morona Santiago.

Zona 7: El Oro, Loja, Zamora Chinchipe.

Zona 8: Guayaquil, Samborondón y Durán (cantones de la Provincia del Guayas).

Zona 9: Distrito Metropolitano de Quito.

2. Distritos: Representan la unidad básica de planificación y prestación de los servicios públicos. Estos se encargan de recoger y reconocer las necesidades de la población en su territorio. Pueden corresponderse con un cantón o con la unión de varios cantones. Actualmente, existen 140 distritos en todo Ecuador.

3. Circuitos: Unidad más pequeña donde se prestan los servicios públicos a nivel nacional. En ellos, adelantan la gestión de las entidades y organismos que conforman la Función Ejecutiva. En cuanto a su extensión, pueden corresponderse con una parroquia o con un grupo de parroquias. Actualmente, existen 1134 circuitos en Ecuador 4 .

\section{Historiografía de la evolución de la salud en Ecuador 1979-2015}

El derecho a la salud de la población ecuatoriana fue reconocido desde hace casi 40 años. Es así que, tras 7 años de dictadura, Ecuador inició un periodo democrático, luego de un referéndum y la aprobación de la Constitución Política del año 1979, que en el numeral 13, artículo 19, sección 1, título 2, definió que los ciudadanos ecuatorianos gozarían de: "el derecho a un nivel de vida que asegure la salud, la alimentación, el vestido, la vivienda, la asistencia médica y los servicios sociales necesarios" ${ }^{\prime 5}$.
Con el paso de los años y a finales de 1996, la República de Ecuador entró en un periodo de inestabilidad democrática que duró 10 años (Tabla 1) el cual generó diversas problemáticas en el área de la salud como: reducción del presupuesto para financiar la prestación de servicios de salud, deterioro en infraestructuras por falta de inversión, mala calidad de los servicios y pérdida de rectoría; este afectó principalmente a la población más pobre, dado que había un cobro directo a los usuarios, asociado al predominio del modelo económico neoliberal6.

Tabla 1. Transición de presidentes del Ecuador 19962007

\begin{tabular}{lll}
\hline \multicolumn{1}{c}{ Presidente } & \multicolumn{2}{c}{ Permanencia en el cargo } \\
\hline Abdalá Bucaram & $\begin{array}{l}\text { Agosto 10 de 1996 - } \\
\text { frtiz }\end{array}$ & 6 meses \\
Fabián Alarcón & Febrero 7 de 1997 de 1997 - & 1 año y 6 \\
Rivera & agosto 10 de 1998 & meses \\
Jamil Mahuad & Agosto 10 de 1998 - & 1 año y 5 \\
Witt & enero 21 de 2000 & meses \\
Gustavo Noboa & Enero 22 de 2000- & 3 años \\
Bejarano & enero 15 de 2003 & 2 años y 3 \\
Lucio Gutiérrez & Enero 15 de 2003 - & meses \\
Borbúa & abril 20 de 2005 & 1 año y 9 \\
Alfredo Palacio & Abril 20 de 2005 - & meses \\
González & enero 15 de 2007 & 10 años y 4 \\
Rafael Correa & Enero 15 de 2007 - & meses \\
Delgado & mayo 24 de 2017 & \\
\hline
\end{tabular}

En este marco político y económico, en 1997 se convocó a consulta popular para elegir una Asamblea Constituyente, la cual tendría como misión redactar la nueva Constitución. Como resultado de este ejercicio democrático se promulgó el Decreto Legislativo No. 000 de agosto de 1998 que dio vida a una nueva Constitución.

Esta nueva carta magna involucró al Estado como garante del derecho a la salud, la promoción y la protección teniendo en cuenta, además, el desarrollo de la seguridad alimentaria, la provisión de agua potable y saneamiento básico y de igual manera, generando el fomento de ambientes saludables en lo familiar, laboral y comunitario, la posibilidad de acceso permanente e ininterrumpido a servicios de salud, conforme a 
los principios de equidad, universalidad, solidaridad, calidad y eficiencia?.

Para dar cumplimiento a lo anterior, a partir de la Constitución de 1998 se expidieron diferentes normas que buscarían garantizar este derecho a la salud, siendo una de las más relevantes, la Ley de Seguridad Social de 2001, publicada en el Registro Oficial $\mathrm{N}^{\circ} 465$, la cual contiene 308 artículos, 23 disposiciones transitorias, una disposición especial única y una disposición general ${ }^{8}$, mediante la cual se establecieron las normas generales de la seguridad social en Ecuador, así como se definieron los principios rectores, los sujetos de protección, los riesgos cubiertos y los recursos que la financiarían. Además, se describen las instituciones que hacen parte del Sistema Nacional de Salud: IESS (Instituto Ecuatoriano de Seguridad Social), ISSFA (Instituto de Seguridad Social de las Fuerzas Armadas), ISSPOL (Instituto de Seguridad Social de la Policía Nacional) y las UMPS (unidades médicas prestadoras de salud), entre otras disposiciones ${ }^{9}$.

Con el propósito de dar cumplimiento a los objetivos del milenio definidos por la Organización de las Naciones Unidas, a cuyo logro se comprometieron todos los países que integran dicha organización, Ecuador creó en 2005 la Secretaría Nacional de los Objetivos del Milenio, la cual, entre sus diversas acciones, impulsó la estrategia del Aseguramiento Universal de Salud en Ecuador, con el objetivo de contribuir al mejoramiento de las condiciones de salud de la población vulnerable de Ecuador ${ }^{10}$.

Sin embargo, a pesar de los diferentes planes y estrategias del gobierno ecuatoriano por garantizar la salud como un derecho fundamental, los resultados en la encuesta de Condiciones de Vida 2005-2006 no fueron los más alentadores en cuanto a la situación de salud y enfermedad de la población ${ }^{11}$. Quizás estos desalentadores resultados fueron producto de la inestabilidad política y de la dolarización de la economía, que impactó en forma desfavorable sobre las condiciones de vida de las familias ecuatorianas.
Por ello, en el periodo de gobierno de transición de Alfredo Palacio, se evidenció la necesidad de actualizar conceptos normativos en temas de salud pública, en derechos humanos y en ciencia y tecnología, por esta razón el Congreso Nacional expidió la Ley Orgánica de Salud, en diciembre de 2006, la cual definió la salud, en el artículo 3, como:

“... el completo estado de bienestar físico, mental y social y no solamente la ausencia de afecciones o enfermedades. Es un derecho humano inalienable, indivisible, irrenunciable e intransigible, cuya protección $y$ garantía es responsabilidad primordial del Estado; y, el resultado de un proceso colectivo de interacción donde Estado, sociedad, familia e individuos convergen para la construcción de ambientes, entornos y estilos de vida saludable."12.

Como se observa el encabezado de la primera línea es coincidente con la declaración en salud establecida como parte de la constitución de la Organización Mundial de la Salud, la cual fue promulgada en 1948, con la finalidad de que existiera cooperación y fomento para la protección de la salud de todos los pueblos ${ }^{13}$.

De igual manera en 2006, el Banco Interamericano de Desarrollo aprobó un préstamo de 90 millones de dólares a Ecuador para apoyar el Proyecto de Aseguramiento Universal de Salud (PRO-AUS), con el fin principal de cubrir a la población más pobre del país. Proyecto que en su primera etapa planteaba mejorar la calidad de los servicios de salud.

Derivado de lo anterior, otro avance importante acaeció en el año 2007, al ser implementado el Programa de Cobertura de Enfermedades Catastróficas, el cual fue financiado con los recursos antes mencionados y ejecutado mediante la Red de Protección Solidaria integrada por el Ministerio de Inclusión Económica y Social (MIES) y el Ministerio de Salud Pública (MSP). Este programa financiaba todos los costos relacionados al tratamiento de 
enfermedades de alto costo en casos seleccionados ${ }^{14}$.

En el año 2006, luego de varios años de crisis política, el candidato a la presidencia, Rafael Correa Delgado como promesa de campaña electoral propuso hacer una nueva constitución, con el objetivo de estabilizar la República de Ecuador y lograr el desarrollo social. Al convertirse en presidente en 2007, dicho proyecto fue presentado ante el Congreso Nacional donde fue aprobado a pesar de que dicho órgano legislativo estaba conformado por una mayoría opositora al nuevo mandatario, fue sometido a consulta popular en abril de 200715 . Tras su aprobación en 2008, se promulga la actual constitución que da el marco político a este país andino. En particular, en los temas sociales, analistas expresan que:

\section{“Esta Constitución desarrolla los derechos sociales y ambientales. Por ejemplo, el derecho al agua (Art. 12), a la alimentación y soberanía alimentaria (Art. 13), la universalización del derecho a la seguridad social (Art. 34), entre otros" 15 .}

En cuanto a salud, los cambios se concentran en el artículo 32 en donde no se limita la salud al buen estado físico y mental de una persona, sino que también incluye otras áreas vinculadas con la política social tales como: servicios públicos, alimentación, educación, trabajo, seguridad social, cultura física, ambientes sanos y todo lo que garantice o mejore las condiciones y la calidad de vida de las personas ${ }^{1}$.

Complementariamente, se destacan los derechos de las personas y grupos de atención prioritaria (Art. 35), se describen y desarrollan con detalle los derechos que atienden las diferencias y condiciones especiales de los miembros de cada uno de los siguientes grupos: adultos mayores, migrantes, mujeres embarazadas, jóvenes, personas con discapacidad, personas con enfermedades catastróficas, privadas de la libertad y las personas usuarias y consumidoras de los servicios públicos ${ }^{15}$.
Como forma de materializar los postulados constitucionales, en el año 2009, la Secretaría Nacional de Planificación y Desarrollo (SENPLADES) elaboró el Plan Nacional de Desarrollo para El Buen Vivir 2009-2013 el cual fue presentado por el Presidente Rafael Correa y aprobado por el Consejo Nacional de Planificación el mismo año. Este Plan es de gran importancia, ya que se fundamenta en tradiciones de los pueblos ancestrales que habitaron y habitan en particular, los Andes ecuatorianos, quienes centran su existir en la armonía con la tierra y en la preponderancia del bienestar del pueblo como colectivo por encima de los intereses particulares de sus integrantes.

Dentro de los objetivos del Plan Nacional de Desarrollo 2009-2013 se destacan cuatro, que se encuentran estrechamente ligados con el derecho a la salud:

1. Auspiciar la igualdad, la cohesión y la integración social y territorial en la diversidad

2. Mejorar las capacidades y potencialidades de la población

3. Aumentar la esperanza y calidad de vida de la población

4. Garantizar los derechos de la naturaleza y promover un ambiente sano y sustentable ${ }^{16}$.

Así, el Plan del Buen Vivir junto con la Agenda Social de Desarrollo Social y los Objetivos de Desarrollo del Milenio se convirtieron en los principales documentos guía de la construcción del Modelo de Atención Integral Familiar, Comunitario e Intercultural de Salud (MAIS-FCI).

El MAIS-FCI define objetivos, políticas y metas prioritarias en salud, constituyéndose en un eje estratégico para el desarrollo y el logro del Buen Vivir. Dicho modelo fue promulgado el 8 de diciembre de 2011, mediante Acuerdo Ministerial; está dirigido al personal de salud de los establecimientos de la Red Pública de Salud y la Red Complementaria, y su implementación es obligatoria en los establecimientos de todos los niveles de atención del Sistema Nacional de Salud (SNS) ${ }^{17}$; cuenta con un amplio marco legal y normativo que busca garantizar el derecho a la salud, la reforma a la estructuración del Sistema Nacional de Salud y la protección de los grupos 
poblacionales. Entre sus principios se destacan la garantía de los derechos de las y los ciudadanos, la universalidad, integralidad, equidad, continuidad, participación social.

Su objetivo es integrar y consolidar la estrategia de la Atención Primaria en Salud (APS) renovada en los tres niveles de atención, reorientando los servicios de salud hacia la promoción de salud y la prevención de enfermedades, fortalecer el proceso de recuperación, rehabilitación de la salud y cuidados paliativos para brindar una atención integral de calidad y profundo respeto a las personas en su diversidad y entorno, con énfasis en la participación organizada de los sujetos sociales. Para ello, el MAIS-FCI tiene cuatro componentes que hacen viable la operación y desarrollo del mismo y son: provisión de servicios, organización, gestión y financiamiento ${ }^{17}$.

Adicional a esto, el gobierno de Rafael Correa, consciente de la alta migración de profesionales de la salud que se dio durante la crisis política, en especial a países como España y Estados Unidos, en Mayo de 2012 impulsó la aprobación del documento "Plan Retorno para Profesionales de la Salud", el cual fue elaborado por la Dirección Nacional de Normatización del Talento Humano y fue dirigido a la mayor cantidad de profesionales de la salud de nacionalidad ecuatoriana que quisieran retornar al Ecuador y a los que se les ofreció beneficios para que aporten los conocimientos adquiridos en el exterior en favor de su país natal. El objetivo de este plan fue eliminar o contrarrestar el déficit existente de médicos y especialistas, como resultado de la combinación de dos factores: la alta emigración de profesionales de la salud en las últimas décadas y el aumento histórico de la demanda de servicios hospitalarios generada por la vigencia de la gratuidad en salud para gran parte de la población ${ }^{18}$.

En este mismo año, como parte de la reestructuración del sector salud, se solicitaron informes de actividades y cumplimiento de objetivos a la Sociedad Ecuatoriana de Medicina Aeronáutica y Espacial, cuya personería jurídica había sido otorgada mediante Acuerdo
Ministerial en el año 1979, y tras cumplirse el plazo estipulado de 15 días sin pronunciamiento al respecto y con la continuidad de su incumplimiento, entró en causal de disolución, derogándose el Acuerdo ministerial que le otorgó personería jurídica ${ }^{19}$.

Otro acontecimiento importante fue la discusión y aprobación del Proyecto de Ley Orgánica Reformatoria a la Ley Orgánica de Salud, Ley 67, donde el Estado ecuatoriano reconoce de interés nacional las enfermedades raras o huérfanas y catastróficas incluyéndose su tratamiento a través de la autoridad sanitaria nacional ${ }^{20}$.

Por otro lado, siendo el Estado el responsable de garantizar la disponibilidad y el acceso a los medicamentos, cuyas características resaltan la calidad, seguridad y eficacia, y además de regular su comercialización y promover la producción nacional y el uso de medicamentos genéricos que resuelvan las necesidades epidemiológicas mediante el acceso estos, teniendo como premisa fundamental el interés por la prevalencia de la salud pública sobre la economía y el comercio; en 2013 se aprobó y publicó la novena revisión del Cuadro Nacional de Medicamentos Básicos, sobre los cuales el Ministerio de Salud Pública con apoyo del Consejo Nacional de Salud debería disponer de las medidas necesarias para garantizar su disponibilidad en el territorio nacional, dado que son los medicamentos esenciales para cubrir las necesidades de salud en la mayoría de la población ${ }^{21}$.

Un buen resumen del enfoque del MAIS lo dio el presidente Rafael Correa en él 2014, en su discurso de inauguración del centro de salud en Ambato, al exponer las características del nuevo modelo de salud, el cual lo describe con un enfoque preventivo más que curativo fundamentándolo de la siguiente forma:

... "Debido a los cambios que se han dado en el perfil patológico de los ecuatorianos, evidenciando que el $80 \%$ de las patologías se pueden resolver en el primer nivel de atención, la mayoría de la población acude a servicios de 
mayor complejidad en los hospitales produciendo una subutilización de dichos servicios y generando la congestión de los mismos" 22 .

De igual manera, este modelo, produjo una descentralización de la atención en salud, lo cual facilitó el acceso a la población en general, llevando servicios como consulta externa y toma de exámenes de laboratorio a los centros de salud, descongestionando los hospitales. Adicionalmente, implementando programas de salud preventiva, familiar y comunitaria. Con esto se pretende resaltar, la atención y la prestación de servicios unificados y de carácter universal centrándolos en la nueva reforma.

\section{Descripción del Sistema de Salud Ecuatoriano}

La segmentación ha sido la característica de la organización del Sistema de Salud en Ecuador, conformado por dos sectores, público y privado; con un sistema de seguridad general financiado por las contribuciones de los trabajadores y los empleadores $^{23}$, la asistencia pública a cargo del Ministerio Público, que no cuenta con ningún tipo de aseguramiento y sistemas privados para los ciudadanos que poseen mayores ingresos y pueden adquirir un seguro.

Esta fragmentación ha tratado de ser atenuada en los últimos años mediante una estrategia encaminada a que los pacientes puedan usar la red de servicios del Seguro Social Ecuatoriano o la de la asistencia pública, indiferente a cuál sea su condición de afiliación. Sin embargo, este proceso aún no ha sido culminado, persistiendo por tanto la fragmentación en el acceso y la atención.

Para comprender mejor la estructura del Sistema de Salud ecuatoriano a continuación, se describen las principales instituciones que lo conforman:

\section{Ministerio de Salud Pública}

El sector público está conformado por diferentes entidades, desde la Ley orgánica se establece al Ministerio de Salud pública como la autoridad sanitaria nacional, siendo el ente rector del sistema de salud, así como garante del derecho a la salud; para lo cual, dicha norma lo faculta para proveer servicios de atención en salud, investigar y articular los actores del sistema. Lo anterior, se refleja en la misión de dicha institución:

"Ejercer la rectoría, regulación, planificación, coordinación, control y gestión de la Salud Pública ecuatoriana a través de la gobernanza y vigilancia y control sanitario y garantizar el derecho a la Salud a través de la provisión de servicios de atención individual, prevención de enfermedades, promoción de la salud e igualdad, la gobernanza de salud, investigación $y$ desarrollo de la ciencia y tecnología; articulación de los actores del sistema, con el fin de garantizar el derecho a la Salud"24.

Lo anterior, fundamentado en el artículo 363 de la Constitución de 2008, en el que se describen entre otras funciones del Ministerio de Salud, la formulación de políticas públicas, la universalización de la atención en salud y el fortalecimiento de los servicios estatales en salud, entre otras.

\section{Instituto Ecuatoriano de Seguridad Social}

Es una entidad, cuya organización y funcionamiento se fundamenta en los principios de solidaridad, obligatoriedad, universalidad, equidad, eficiencia, subsidiariedad y suficiencia. Se encarga de aplicar el Sistema del Seguro General Obligatorio que forma parte del sistema nacional de Seguridad Social. Fue creado mediante el Decreto Supremo № 40 del 25 de Julio de 1970 y publicado en el Registro Oficial № 15 del 10 de Julio de 1970.

La constitución de 2008 plasma en el artículo 369 , el seguro universal obligatorio de la siguiente forma:

"El seguro universal obligatorio cubrirá las contingencias de 
enfermedad, maternidad, paternidad, riesgos de trabajo, cesantía, desempleo, vejez, invalidez, discapacidad, muerte y aquellas que defina la Ley. Las prestaciones de salud de las contingencias de enfermedad y maternidad se brindarán a través de la red pública integral de salud"1.

Lo anterior, con el fin de entender el artículo 370 de dicha constitución, el cual relaciona al Instituto Ecuatoriano de Seguridad Social (IESS) como una entidad autónoma, responsable de la prestación de las contingencias del seguro universal obligatorio a sus afiliados. El IESS, atiende mediante afiliación personal a los trabajadores formales (el $28 \%$ de la población económicamente activa) y al sector rural, mediante el seguro social campesino.

\section{Seguro Social Campesino}

En la constitución de 2008, se define en el artículo 373, el Seguro Social Campesino, como parte del IESS, siendo este un régimen especial, de seguro universal obligatorio. De la misma manera establece como sus afiliados a la población rural y a las personas dedicadas a la pesca artesanal. La financiación de este seguro se hace con el aporte solidario de las personas aseguradas y empleadoras del sistema nacional de seguridad social, el aporte diferenciado de jefas o jefes de las familias protegidas y con las asignaciones fiscales para que, entre todas las fuentes de financiación, garanticen el fortalecimiento y desarrollo de dicho seguro ${ }^{1}$.

\section{Instituto de Seguridad Social de las Fuerzas Armadas del Ecuador (ISSFA)}

Existen regímenes especiales, dentro del Sistema de Salud Ecuatoriano, como el Servicio de Sanidad de las Fuerzas Armadas y el Servicio de Sanidad de la Policía, dependientes de los institutos de seguridad social de las respectivas instancias. Las Fuerzas Armadas y la Policía disponen de servicios ambulatorios y de hospitalización para sus miembros y familiares y funcionan bajo la modalidad de seguro de salud.

\begin{abstract}
Artículo 370. “...La policía nacional y las fuerzas armadas podrán contar con un régimen especial de seguridad social, de acuerdo con la ley; sus entidades de seguridad social formarán parte de la red pública integral de salud y del sistema de seguridad social"1.
\end{abstract}

En 1992, la Ley de Seguridad Social de las Fuerzas Armadas, estableció en el artículo $1^{\circ}$, que el ISSFA es un organismo autónomo, con finalidad social, con personería jurídica, patrimonio propio, domiciliado en la ciudad de Quito, y no está sujeto a la intervención de la Contraloría General del Estado.

\section{Junta de Beneficencia de Guayaquil (JBG) y la Sociedad Protectora de la Infancia de Guayaquil}

Estas dos instituciones son entidades privadas, sin ánimo de lucro, atienden la recuperación de la salud de población de medianos y escasos recursos, principalmente de la región de la Costa $^{25}$.

\section{Estructuras de prestación de servicios de salud}

En el sector público, el Ministerio de Salud Pública es quien concentra la mayor cantidad de establecimientos de salud, principalmente con establecimientos del primer nivel con cobertura tanto urbana como rural entre los que se encuentran los establecimientos del Seguro Social Campesino y los del IESS (Tabla 2).

También se destacan los establecimientos privados con un número similar a los del Ministerio de Salud Pública. Cabe resaltar que un número importante de estos figuran anexos al IESS y su característica principal es que son consultorios privados acreditados por esta institución para brindar atención a la población de su responsabilidad 26 . 
Tabla 2. Número de establecimientos de salud por institución

\begin{tabular}{lrrr}
\hline \multicolumn{1}{c}{ Establecimientos de salud } & $\mathbf{2 0 0 0}$ & $\mathbf{2 0 0 6}$ & $\mathbf{2 0 1 0}$ \\
\hline Ministerio de Salud Pública & 1.685 & 1.737 & 1.922 \\
$\begin{array}{l}\text { Instituto de Seguridad Social } \\
\text { (IESS) }\end{array}$ & 85 & 51 & 72 \\
Seguro Social Campesino & 577 & 577 & 606 \\
Municipios & 38 & 38 & 75 \\
Ministerio de Defensa Nacional & 82 & 66 & 73 \\
Ministerio de Justicia y de & 41 & 36 & 36 \\
Gobierno y Policía & 98 & 97 & 92 \\
Ministerio de Educación & 555 & 682 & 760 \\
Privados & 5 & 3 & 5 \\
Junta de Beneficencia de & 7 & 12 & 13 \\
Guayaquil & & & \\
Sociedad Lucha con el Cáncer & 352 & 294 & 257 \\
(SOLCA) & 71 & 88 & 70 \\
Anexos al IESS & 3.596 & 3681 & 3.981 \\
\hline Otros* & & & \\
Total de establecimientos & & & \\
\hline Fuente: INEC, Estadísticas de recursos y actividades de salud - ERAS, 2000, \\
2006, 2010.
\end{tabular}

Como se observa en la Tabla 2, para el año 2010 en Ecuador existían 3981 establecimientos de salud, entre públicos y privados, lo que representó un incremento del $10 \%$ respecto al número de establecimientos existente en el año 2000. Este dato toma mayor relevancia cuando se observa que este incremento se dio principalmente, por un aumento en la oferta pública, lo que denota la intencionalidad del gobierno de garantizar de forma directa la atención en salud a sus ciudadanos y, por ende, restringiendo el campo de acción de los particulares.

Adicionalmente, la Secretaría Nacional de Planificación y Desarrollo (SENPLADES) en su informe de rendición en cuentas 2014 refleja que, en cuanto a salud, se han hecho mejoras en 60 hospitales y 85 centros de salud generando aumento en la accesibilidad a servicios de salud y calidad de la atención ${ }^{27}$.

Por su parte, los resultados de la Encuesta de Condiciones de Vida 1999, 2006 y 2014 (Tabla 3 ), evidencia que el período de inestabilidad democrática afectó la cobertura en salud, en la cual se observa una disminución en el porcentaje de afiliación al IESS entre el período 1999 a 2006; en contraste con el aumento de la adquisición de seguros privados, en el mismo período. Sin embargo, en el período comprendido de 2006 a 2014, que corresponde al mandato presidencial de Rafael Correa Delgado, se puede destacar que la cobertura en salud da un giro importante, mostrando un aumento del 17.2 puntos porcentuales en la afiliación al IESS, que a su vez generó una disminución de los servicios de salud privados. Estos resultados muestran los esfuerzos del gobierno en esta última década, para lograr una mayor cobertura del aseguramiento público en salud para el país.

Tabla 3. Cobertura Sistema de Salud Ecuador

\begin{tabular}{lrrr}
\hline & 1999 & $\mathbf{2 0 0 6}$ & $\mathbf{2 0 1 4}$ \\
\hline Afiliación al IESS & 25,7 & 21,7 & 38,9 \\
Seguro privado & 3,9 & 6,6 & 4,0 \\
& & & \\
\hline
\end{tabular}

Fuente. INEC. Encuesta Condiciones de Vida 1999, 2006, 2014

De la misma manera el IESS, presenta la Información estadística de las Direcciones Nacionales y Seguros Especializados, destacándose, un incremento del 105\% de afiliados activos en el Seguro General Obligatorio en el período 2007- 2014. Mientras que la cobertura del Seguro Social Campesino creció al $68 \%$ durante el mismo período de análisis. Para diciembre 2014, las provincias que concentran mayor número de afiliados activos son: Pichincha (35\% del total), Guayas (27\%) y Azuay $(6 \%)^{28}$.

\section{Discusión}

El devenir histórico del Sistema de Salud de Ecuador en el periodo comprendido entre 1979 y 2014 es el reflejo de los acontecimientos políticos y económicos que acaecieron tanto en el ámbito global como en el regional y local.

La constitución de 1979 y su aceptación de la salud como derecho fundamental se enmarca en las aspiraciones democráticas emergidas con el fin de la dictadura. Las dictaduras fueron las 
formas de gobierno preeminentes en Latinoamérica en los años 70 e inicios de los 80 y se correspondían con el temor norteamericano del avance del modelo comunista impulsado por la Unión Soviética en Latinoamérica a través del gobierno de Cuba, a partir del triunfo revolucionario logrado en dicha isla en 1959. Este modelo dictatorial suprimía los derechos fundamentales de los ciudadanos y reprimía cualquier acción que fuera en contravía de los intereses norteamericanos ${ }^{29}$. En este sentido, la dictadura ecuatoriana puede ser comprendida desde las lógicas impuestas por el Departamento de Estado norteamericano en el marco de la denominada Operación Cóndor, la cual guío las dictaduras de Chile, Argentina, Brasil, Uruguay y Paraguay 30 .

En ese contexto la salud era entendida en buena medida como una acción caritativa, que se enmarcaba en la política instituida por el presidente John F. Kennedy denominada como La Alianza para el Progreso, la cual buscaba que acciones conjuntas entre Estados Unidos de Norteamérica y los gobiernos locales cubrieran las necesidades de los más pobres, no con una intención basada en derechos humanos sino como una forma de control político y social sobre poblaciones que pudiesen ser víctimas del germen comunista, como lo denominaría, posteriormente, el secretario de Estado norteamericano Henry Kissinger ${ }^{29-31}$.

Finalizada la dictadura se instituye el sueño democrático, el cual trató de desarrollarse bajo el modelo proteccionista y de sustitución de importaciones que había sido planteado desde la década de los 60 por la Comisión Económica para Latinoamérica (CEPAL), ideas que planteó Raúl Prebisch con base en el modelo de intervención del Estado postulado por John Maynard Keynes. Sin embargo, este modelo culminaría su implementación a causa de dos factores: el primero, el modelo proteccionista creó monopolios y oligopolios nacionales que impidieron el desarrollo de mercados internos y el segundo, la crisis de la deuda externa latinoamericana que condujo a la cesación de pagos de los créditos tomados por estos países procedentes de las denominadas instituciones de Breton Woods ${ }^{31}$.

Esto llevó a que las instituciones financieras multilaterales, Fondo Monetario Internacional y Banco Mundial, junto con la Reserva Federal de Estados Unidos, impusieran a los países latinoamericanos el denominado Consenso de Washington, como requisito para que pudiesen acceder nuevamente al crédito ${ }^{32,33}$. Este consenso no era más que la llegada del modelo neoliberal creado, principalmente, por la escuela de la Universidad de Chicago, liderada por Milton Friedman ${ }^{34}$, a Latinoamérica, lo que implicó para el sector salud la privatización tanto del aseguramiento como de la prestación de los servicios de salud, con el fin de reducir el gasto en salud de los países, liberando de esta forma recursos que les permitiesen pagar las deudas adquiridas con las instituciones multilaterales de crédito ${ }^{32}$.

Es en este marco que emerge en Ecuador la constitución de 1998, que trajo como resultado una profunda inestabilidad política al país; fruto del inconformismo social en especial por parte de grupos indígenas y de trabajadores que vieron mermada su condición de vida, la cual se vio agravada por la decisión de dolarizar la economía en 2000, afectando la industria nacional, reduciendo su competitividad y por ende, elevándose el desempleo. Esto aunado a que en Ecuador las crisis económicas han agudizado las crisis políticas, en buena medida, asociadas a la ausencia de una institucionalidad lo suficientemente estable que oriente los esfuerzos de los distintos actores a la solución de los problemas, hizo que el sistema político colapsara ${ }^{35}$.

En lo que atañe a salud, el modelo neoliberal impulsó la separación de las acciones individuales de las colectivas, transformando a las primeras en objeto de las fuerzas del mercado y por ende, privatizando su provisión, hecho que impulsó el surgimiento de infraestructuras de salud privadas y modelos de seguros en salud como la medicina prepagada, a los cuales asistían quienes pudieran pagar por ello, mientras que la atención de quienes no tenían el poder 
adquisitivo para costear por su atención en salud era provista por infraestructuras públicas de deficiente calidad dadas las restricciones financieras que desde el gobierno se les imponían. Por su parte, las denominadas acciones colectivas, relacionadas con los determinantes sociales de la salud, al no poder ser objeto de lucro, su costeo se convirtió en responsabilidad del Estado. Esto incrementó la inequidad en el acceso a la salud y profundizó la segregación social y la fragmentación en la atención ${ }^{36}$.

Dentro de este contexto surge un movimiento cívico que se acerca en su filosofía política a la denominada nueva izquierda latinoamericana, cuyo principal antecedente es la elección de Hugo Chávez como presidente de Venezuela, la cual busca la reivindicación social de aquellos seres humanos que han quedado al margen de la economía de mercado, así como un alto componente de revalorización de lo nacional, ideología denominada por el mismo Chávez como el Socialismo del Siglo XXI. Así, llega al poder en Ecuador Rafael Correa, quien con la base popular iniciará un camino de transformación del modelo social y económico del país ${ }^{35,37}$.

Como base de la transformación, emergerá la Constitución de 2008 y su correlato el plan de gobierno denominado del Buen Vivir, el cual recupera la cosmovisión ancestral de los pueblos andinos basada en respeto a la madre tierra y valoración del bienestar colectivo como forma de construir la fortaleza del Estado y de lo público para establecer el camino hacia un desarrollo incluyente que parta de garantizar los derechos fundamentales a los ciudadanos ${ }^{16,37}$. Para el caso de la salud, esto se reflejó en un fortalecimiento de lo público mediante la formulación del MAISFCI el cual guió las acciones encaminadas a intervenir sobre determinantes sociales de la salud como alimentación, agua potable y vivienda. De igual forma, orientó la construcción y recuperación de la infraestructura pública de salud con el fin de garantizar el acceso oportuno a los servicios a la población y estableció políticas de acceso a medicamentos y de repatriación de talento humano migrado al exterior con el fin de cerrar la brecha social histórica existente en Ecuador ${ }^{17}$.

La política del MAIS-FCI también puede entenderse como un reflejo del relanzamiento de la Atención Primaria en Salud renovada realizada por la Organización Mundial de la Salud en 2008, la cual rescata el enfoque preventivo, la transectorialidad y el fortalecimiento de las redes de prestación de salud como principales estrategias para la garantía del derecho a la salud de los pueblos ${ }^{38}$.

El establecimiento de estas políticas no ha sido fácil ya que ha chocado con los intereses particulares de las multinacionales farmacéuticas ${ }^{39}$, en el caso de los medicamentos, de los dueños de hospitales privados y de profesionales de salud que ejercen de manera independiente, para el caso de la prestación de servicios de salud, lo que ha ralentizado los avances. Sin embargo, a pesar de esta resistencia, el adelanto en el Sistema de Salud ecuatoriano ha sido evidente, lo que ha hecho que la población lo use más y obtenga beneficios que antes no recibía de él.

Finalmente, se puede afirmar que si bien aún no se ha logrado garantizar el derecho a la salud plenamente a todos los ecuatorianos, los esfuerzos realizados por el gobierno de dicho país, en especial desde 2006, han generado grandes avances en pro de alcanzar tan loable meta, con lo que se honraría la frase que enmarca el Plan del Buen Vivir: Ecuador ama la vida.

\section{Conclusiones}

Los cambios en el Sistema de Salud de Ecuador pueden comprenderse como el resultado de la confluencia de dos grandes factores asociados al devenir histórico de esta nación: el primero, las macro tendencias políticas y económicas impartidas desde los centros de poder mundial, en especial, Estados Unidos y en segundo término, la adopción de las mismas por parte de los gobiernos de las décadas de los 70, 80 y 90, que crearon el ambiente propicio para la reacción popular que derivó en la consolidación 
de un gobierno originado en un movimiento cívico que se apropió de su realidad y de la orientación de su política social y económica, enmarcado en el denominado Socialismo del Siglo XXI.

Ecuador se convierte así en un país cuyo Sistema de Salud amerita ser estudiado y reflexionado desde contextos amplios, que permitan comprender las dinámicas que lo han convertido en un ejemplo de fortalecimiento del sector público, como forma preeminente de garantizar el derecho a la salud a todos los ciudadanos.

Conflicto de intereses: Ninguno declarado.

\section{Agradecimientos}

Este artículo se realizó en investigación conjunta entre la Universidad Jorge Tadeo Lozano, Bogotá, Colombia y la ESPAE Graduate School of Management, de la Escuela Superior Politécnica del Litoral, Guayaquil, Ecuador.

\section{Referencias}

1. Constitución de la República de Ecuador. Registro Oficial $\mathrm{N}^{\circ} 449$, (20 octubre 2008).

2. Instituto Nacional de Estadísticas y Censos, INEC. Compendio Estadístico de 2013. Ecuador: INEC; 2013. Disponible http://www.ecuadorencifras.gob.ec/compendioestadistico/

3. Secretaría Nacional de Planificación y Desarrollo, SENPLADES. Niveles Administrativos De Planificación. Quito: SENPLADES; 2012 [actualizado s.f.; citado 28 julio 2015]. Disponible en: http://www.planificación.gob.ec/3-nivelesadministrativos-de-planificacion.

4. Instituto Nacional de estadísticos y Censos, INEC. División Político Administrativa El Ecuador. Quito: INEC; s.f. Disponible en http://www.ecuadorencifras.gob.ec/division-politicoadministrativa-el-ecuador/

5. Constitución de la República de Ecuador. Registro Oficial $N^{\circ} 800$, (27 marzo 1979)

6. Malo-Serrano, M; Malo-Corral, N. Reforma de Salud en Ecuador: Nunca más el derecho a la salud como un privilegio. Revista Peruana de Medicina Experimental y de Salud Pública. 2014; 31(4):754-61.

7. Ley 000. Registro Oficial $\mathrm{N}^{\circ} 144$, (18 agosto 2000).

8. Instituto Ecuatoriano de Seguridad Social, IESS. ¿Quiénes somos? Quito: IESS; 1970 [actualizado s.f.; citado 9 junio 2015]. Disponible en: http://www.iess.gob.ec/es/web/guest/inst-quienessomos.

9. Ley de Seguridad Social. Registro oficial $N^{\circ} 465$, (30 noviembre 2001).

10. Lucio, R.; Villacrés, N.; et al. Sistemas de Salud de Ecuador. Salud Pública de México. 2011; vol. 53, suplemento 2 de 2011.

11. Instituto Nacional de Estadística y Censos, INEC; Secretaria Nacional de Planificación y Desarrollo, SENPLADES. Encuesta de Condiciones de Vida 2006. Ecuador: INEC, SENPLADES; 2006. [Actualizado 2014; citado 29 julio 2015]. Disponible en http://anda.inec.gob.ec/anda/index.php/catalog/358

12. Ley Orgánica de Salud. Registro Oficial N ${ }^{\circ} 423,(22$ de diciembre de 2006)

13. Organización Mundial de la Salud, OMS. ¿Cómo define la OMS la salud?. Nueva York: OMS; 1948. [Actualizado 2015; Consultado 15 mayo 2015]. Disponible en http://www.who.int/suggestions/faq/es/

14. Organización Panamericana de la Salud, OPS. Salud en las Américas, Edición de 2012: Volumen de países. Washington D.C.: OPS; 2012. [Actualizado s.f.; citado 15 mayo 2015]. Disponible en http://www.paho.org/saludenlasamericas/index.php? option=com_docman\&task=doc_view\&gid=202\&Itemid $=$

15. Corte Constitucional de Ecuador. La Constitución del Ecuador cumple 7 años hoy 20 de octubre de 2015. Ecuador: Corte Constitucional de Ecuador; 2015. Disponible en https://www.corteconstitucional.gob.ec/index.php/bol etines-de-prensa/item/1685-la-constituci\%C3\%B3ndel-ecuador-cumple-7-a\%C3\%B1os-hoy-20-deoctubre-de-2015.html

16. Secretaría Nacional de Planificación y Desarrollo, SENPLADES. Plan Nacional de Desarrollo, Plan Nacional para El Buen Vivir 2009-2013: Construyendo un Estado Plurinacional e Intercultural (Segunda edición). Quito: SENPLADES; 2009. [Actualizado: s.f., Citado: 13 diciembre 2015]. Disponible en http://www.planificacion.gob.ec/wpcontent/uploads/downloads/2012/07/Plan_Nacional_ para_el_Buen_Vivir.pdf

17. Ministerio de Salud Pública del Ecuador, Subsecretaría Nacional de Gobernanza de la Salud Pública, Dirección Nacional de Articulación y Manejo del Sistema Nacional de Salud y de la Red Pública. Modelo de Atención Integral del Sistema Nacional de Salud. Quito; Dirección Nacional de Articulación y Manejo del Sistema Nacional de Salud y la Red pública, 2012. [Actualizado s.f.; citado 10 octubre 2015]. Disponible en

http://instituciones.msp.gob.ec/somossalud/images/d ocumentos/guia/Manual_MAIS-MSP12.12.12.pdf

18. Dirección Nacional de Normatización de Talento Humano. Plan Retorno para Profesionales de la Salud. Ecuador: Dirección Nacional de Normatización de Talento Humano; 2012. Disponible en http://fase4.reterirva.it/DOCRIRVA/all.9plan_retorno\%20Salud[1].pdf 
19. Ministerio de Salud Pública. Carina Vance Mafla. Ministra de Salud Pública (E). Acuerdo Ministerial No. 1041 del 29 de mayo de 2012. Disponible en http://www.derechoecuador.com/productos/product o/catalogo/registrosficiales/2012/julio/code/20366/r egistro-oficial-no-742--martes10-de-julio-del2012\#1041

20. Asamblea Nacional República del Ecuador. Registro Oficial № 625. Quito: Asamblea Nacional; 2012.

21. Ministerio de Salud Pública. Carina Vance Mafla. Ministra de Salud Pública. Acuerdo Ministerial No. 00004288. QUITO: Ministerio de Salud Pública; 2013.

22. Presidencia de la República del Ecuador, SECOM. Discurso Presidente Inauguración del Centro de Salud tipo A de Ambatillo - Ambato [Video]. YouTube: Ambato, Ecuador; 2014.

23. González-Andrade, López-Pulles. Ecuador: Public Health Genomics. Public Health 172 Genomics 2010;13:171-180.

24. Ministerio de Salud Pública. Valores/Misión/Visión. Ecuador: Ministerio de salud Pública; s.f. Disponible en http://www.salud.gob.ec/valores-mision-vision/

25. Comisión Económica para América Latina y el Caribe, CEPAL. Seguridad Social (Salud) Ecuador. Ecuador: CEPAL, s.f. Disponible en http://www.cepal.org/ilpes/noticias/paginas/3/1311 3/salud_ecuador.ppt

26. Ministerio de Salud Pública. Datos esenciales de Salud: Una mirada a la década 2000-2010. Ecuador; Ministerio de Salud Pública, 2012. [Actualizado s.f.; citado 30 junio 2015] Disponible en http://www.salud.gob.ec/wpcontent/uploads/downloads/2013/05/Datosesenciales-de-salud-2000-2010.pdf

27. Secretaria Nacional de Planificación y Desarrollo. Rendición de cuentas. 2014. Disponible en http://www.planificacion.gob.ec/wpcontent/uploads/downloads/2015/02/Informerendici\%C3\%B3n-de-cuentas-Zona-9-.pdf

28. Instituto Ecuatoriano de Seguridad Social. Informe De Rendición De Cuentas. 2014. Disponible en https://www.iess.gob.ec/documents/10162/3780216 /2015+04+01+Rendicion+de+cuentas+v3.pdf

29. Chomsky N. ¿Quién domina el mundo? Barcelona: Ediciones B, S.A.; 2016.

30. Barreto Velázquez N. La Operación Cóndor: un enfoque comparativo. Histórica (02528894) [serial on the Internet]. (2013, Dec), [cited March 28, 2017]; 37(2): 171-178. Available from: Fuente Académica Premier.

31. Gabay R. Revisitando a Raúl Prebisch y al papel de la CEPAL en las ciencias sociales de América Latina. Íconos. Revista De Ciencias Sociales [serial on the Internet]. (2008, May), [cited March 28, 2017]; (31): 103-113. Available from: Fuente Académica Premier.

32. Wilson Giovanni J. El derecho a la salud una búsqueda inacabada para la sociedad colombiana. Revista EAN [serial on the Internet]. (2009), [cited March 14, 2017]; (67): 107. Available from: SciELO.

33. López Meraz Ó, Villegas Loeza D. Hegemonía selectiva en América Latina: puntos neoliberales de encuentro entre México y Colombia. Espacio Abierto. Cuaderno
Venezolano De Sociología [serial on the Internet]. (2015, Apr), [cited March 28, 2017]; 24(2): 207-222. Available from: Fuente Académica Premier.

34. Ebenstein L. The Increasingly Libertarian Milton Friedman: An Ideological Profile. Econ Journal Watch [serial on the Internet]. (2014, Jan), [cited March 28, 2017]; 11(1): 81-96. Available from: Business Source Complete.

35. Ulloa C. Emergencia de populismo y ruptura de acuerdos políticos. Revista De Estudios Sociales [serial on the Internet]. (2016, Apr), [cited March 28, 2017]; (56):39-50. Available from: Fuente Académica Premier.

36. Hernández Gómez Lidia. Neoliberalismo y salud, qué pasó en América Latina, la experiencia cubana. Rev Cubana Salud Pública [Internet]. 2002 Dec [cited 2017 Mar 28]; 28(3):. Available from: http://scielo.sld.cu/scielo.php?script=sci_arttext\&pid= S0864-34662002000300007\&lng=en.

37. Manosalvas M. Buen vivir o sumak kawsay. En busca de nuevos referenciales para la acción pública en Ecuador. Íconos. Revista De Ciencias Sociales [serial on the Internet]. (2014, May), [cited March 28, 2017]; (49): 101-121.

38. Organización Panamericana de la Salud. Renovación de la atención primaria de salud en las Américas: documento de posición de la Organización Panamericana de la Salud/Organización Mundial de la Salud (OPS/OMS). Disponible en https://cursos.campusvirtualsp.org/file.php/118/Mod ulo_I/md3-lp-renovacion-APS-spa-2007.pdf

39. Arbeláez M. Impatentabilidad De Medicamentos Esenciales. Propiedad Inmaterial [serial on the Internet]. (2014, Jan), [cited March 28, 2017]; (18):5584. Available from: Fuente Académica Premier. 